\title{
Calculation method of evaluators' influence based on group order relation analysis method in bargaining evaluation
}

\author{
Qiang Mao ${ }^{a^{*}}$, Anye Liu ${ }^{b}$ and Yue Sun ${ }^{\mathrm{c}}$ \\ School of Management, Bohai University, Jinzhou 121013, China.

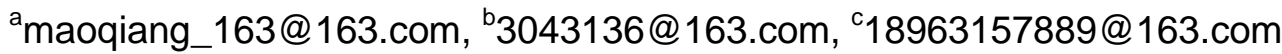

\begin{abstract}
Keywords: Bargaining evaluation; Evaluators' influence; Order relation analysis method; Level weight
\end{abstract}

\begin{abstract}
For the limitations about the calculation method of evaluators' influence in existing bargaining evaluation, a calculation method of evaluators' influence based on order relation analysis method in bargaining evaluation is proposed. The traditional group relation analysis method is improved in the paper, which is according to the level weight based on evaluators' professional and technical title. The credibility of evaluation conclusion can be judged from evaluators' influence by people. Finally, a numerical example is given to illustrate the simplicity and maneuverability of the proposed method.
\end{abstract}

\section{Introduction}

Many scholars are attracted to the research of comprehensive evaluation for its widely application background in engineering design and economic management fields. Guo(1993) introduced the concept of bargaining evaluation, which supposes that the evaluation objects and evaluation index in the evaluation system are assured, and the different opinions given by evaluators just focus on index weight [1]. In recent years, a wealth of research findings can be found in group evaluation field [1-8]. Liu et al. (2005) hold on the idea that determining the weight of each attribute was the key to solve bargaining evaluation problem, and they proposed a method of determining attribute weight based on ordered weighted averaging operator for bargaining evaluation problem [2]. On the basis of literatures, Li et al. (2012) proposed an objective self-determining evaluation method based on collaborative perspective, which can be used to solve interval number comprehensive evaluation problem [3].

Above mentioned literatures are the foundations of bargaining evaluation theory, but they hardly consider evaluators' influence, and the influence of the evaluators is objective existence for they have different background. Chen et al. (2006) proposed an approach to judge each expert's influence by consistency degree of each alternative and index [4]. On the basis of literature, Chen et al. (2007) proposed a ranking approach to assessment level of experts based on total factor score of expert [5]. $\mathrm{Xu}$ et al. (2010) proposes an approach to determine experts' influence based on the degree of difference between individual and group decision matrix [6]. Zhang et al. (2010) proposed a method of group bargaining evaluation based on the Monte Carlo simulation, and evaluators' influence can be obtained on the basis of the proximity of their opinions [7].

The literatures from [4] to [7] proposed some useful approaches to obtain evaluators' influence in group evaluation problem, but the evaluators' influence is assured by consistency degree, and it doesn't mean the conclusion is reliable. In order to provide reference for the credibility of evaluation findings and the choice of evaluator in practical application, Mao et al. $(2013,2004)$ proposed a calculation method of evaluators' authority in bargaining evaluation [8,9]. But the method given by literature $[8,9]$ is limited by assumptions, it still has something to improve. Based on this, this paper proposes a method based on order relation analysis method to calculate evaluators' influence. The method not only can help evaluation demander choose proper scale evaluators for evaluation problem, but also help public judge the credibility of evaluation conclusion based on the evaluators' influence. At last, the simplicity and feasibility of the method is verified by a numerical example. 


\section{Problem description}

Classic bargaining evaluation method is based on a multi-index evaluation system with evaluation object $O=\left(o_{1}, o_{2}, \cdots, o_{n}\right)$ and evaluation index $X=\left(x_{1}, x_{2}, \cdots, x_{m}\right)$. The index observed value $x_{i j}=x_{j}\left(o_{i}\right)$ $(i=1,2, \cdots, n ; j=1,2, \cdots, m)$ is objective existence, $y_{i}$ is evaluation value of $o_{i}$, and the credible degree of comprehensive evaluation results is directly relates to the index weight. $\mathrm{D}_{j, k}=\left[\lambda_{j}^{k \mathrm{~L}}, \lambda_{j}^{k \mathrm{U}}\right]$ is named as a bargain interval for evaluation index $x_{j}$ which is given by evaluator $e_{k}$, and $\lambda_{j}^{k \mathrm{~L}}$ is named as lower bound value which is the minimum value that $\lambda_{j}$ can be obtained, while $\lambda_{j}{ }^{k \mathrm{U}}$ is named as upper bound value which is the maximum value that $\lambda_{j}$ can be obtained, then $0 \leqslant \lambda_{j}^{k \mathrm{~L}} \leqslant \lambda_{j}^{k \mathrm{U}} \leqslant 1$ can be known. Let $\mathrm{e}\left(\mathrm{D}_{j, k}\right)=\lambda_{j}^{k \mathrm{U}}-\lambda_{j}^{k \mathrm{~L}}$, and $\mathrm{e}\left(\mathrm{D}_{j, k}\right)$ is named as interval width. Let $\mathrm{n}\left(\mathrm{D}_{j, k}\right)=\left(\lambda_{j}^{k \mathrm{U}}-\lambda_{j}^{k \mathrm{~L}}\right) / 2$, and $\mathrm{n}\left(\mathrm{D}_{j, k}\right)$ is named as interval midpoint. Evaluators' different opinions about index weight will reach an agreement through bargaining. Now the question is that how to determine the bargaining weights can ensure the credibility of evaluation results.

\section{Principle and method}

Section A, the determination method of evaluators' influence. Influence is a kind of ability that can change others' ideas and make them to move. This paper use influence to describe each evaluator's influence of the evaluation conclusion. Due to the evaluators are belonging to the same area, they know each other very well, so a calculation method of evaluator's influence is given which is referred to the method named rank correlation analysis method I (G1-) in literature[10].

At first, obtain the influence sorting of evaluation group under the view of every evaluator. The steps can be listed as follows:

Step 1: Let evaluator $e_{k}(k=1,2, \ldots, L)$ pick up the most influential evaluator in evaluation group $E=\left(e_{1}, e_{2}, \cdots, e_{L}\right)$, the evaluator who is picked by evaluator $e_{k}$ called $e_{1}^{\left(e_{k}\right)}$.

Step 2: Let evaluator $e_{k}$ pick up the most influential evaluator in rest of evaluation group $E$ in which there are $L-1$ evaluators, and the evaluator who is picked by evaluator $e_{k}$ called $e_{2}^{\left(e_{k}\right)}$.

;

Step $h$ : Let evaluator $e_{k}$ pick up the most influential evaluator in rest of evaluation group $\boldsymbol{E}$ in which there are $L-(h-1)$ evaluators, and the evaluator who is picked by evaluator $e_{k}$ called $e_{h}^{\left(e_{k}\right)}$ $(h=2,3, \ldots, L)$.

Step $L$ : After $L-1$ round, the last evaluator called $e_{L}^{\left(e_{k}\right)}$.

Then, the influence sorting $\eta_{1}^{\left(\mathrm{e}_{k}\right)} \succ \eta_{2}^{\left(\mathrm{e}_{k}\right)} \succ \cdots \succ \eta_{L}^{\left(\mathrm{e}_{k}\right)}$ can be obtained under the view of evaluator $e_{k}$.

Secondly, compare $\eta_{h-1}^{\left(\mathrm{e}_{k}\right)}$ with $\eta_{h}^{\left(\mathrm{e}_{k}\right)}$.

Let $\eta_{h-1}^{\left(\mathrm{e}_{k}\right)} / \eta_{h}^{\left(\mathrm{e}_{k}\right)}=r_{h}^{\left(\mathrm{e}_{k}\right)}, r_{h}^{\left(\mathrm{e}_{k}\right)}$ is called the ratio of influence under the view of evaluator $e_{k}$.

Theorem 1[10] If $\eta_{h-1}^{\left(\mathrm{e}_{k}\right)} / \eta_{h}^{\left(\mathrm{e}_{k}\right)}=r_{h}^{\left(\mathrm{e}_{k}\right)}$, and the influence relationship among evaluators satisfy the condition that $\eta_{1}^{\left(\mathrm{e}_{k}\right)} \succ \eta_{2}^{\left(\mathrm{e}_{k}\right)} \succ \cdots \succ \eta_{L}^{\left(\mathrm{e}_{k}\right)}$, then

$$
\eta_{L}^{\left(\mathrm{e}_{k}\right)}=\left(1+\sum_{k=2}^{L} \prod_{h=k}^{L} r_{h}^{\left(\mathrm{e}_{k}\right)}\right)^{-1}
$$

The G1- method given by literature [10] supposes that evaluators' influence have no difference, but in the real life people always have different ability, so the paper give a method to calculate evaluators' influence based on their technical titles. And without loss of generality, the paper supposes the number of technical titles is an inverse index. Let $w_{e_{k}}=\left(\mathrm{u}+1-\mathrm{v}_{e_{k}}\right) / \mathrm{u}$ which is named as position weight which is a factor based on their technical titles for calculating the evaluators influence, and the $\mathrm{u}$ is total level number of technical titles in the field, while the $v_{e_{k}}$ is the level number of 
evaluator $e_{k}$. Let $\eta_{k}=w_{e_{1}} \eta_{k}^{\left(e_{1}\right)}+w_{e_{2}} \eta_{k}^{\left(e_{2}\right)}+\cdots+w_{e_{L}} \eta_{k}^{\left(e_{L}\right)}$, then we can obtain the every evaluator's influence.

Section B, the determination method of index bargaining weight. The determination method of index bargaining weight is inspired by the thought of literature [10], and three kinds of circumstances were considered in the paper, then the specific methods are as follows.

1) For any $\mathrm{D}_{j, k}$, if $\lambda_{j, k}^{\mathrm{D}}=\lambda_{j, k} \mathrm{U}=\lambda_{j, k}$, then $\lambda_{j}^{\prime}=\sum_{k=1}^{L} \eta_{k} \lambda_{j, k}, j=1,2, \ldots, m$.

2) For any $\mathrm{D}_{j, k}$, if $\lambda_{j, k} \mathrm{D}_{\neq} \neq \lambda_{j, k} \mathrm{U}$, then

$$
\lambda_{j}^{\prime}=\left(\sum_{k=1}^{L} \frac{\eta_{k}\left(\lambda_{j, k}{ }^{D}+\lambda_{j, k}{ }^{U}\right)}{2\left(\lambda_{j, k}{ }^{U}-\lambda_{j, k}{ }^{D}\right)}\right) /\left(\sum_{k=1}^{L} \frac{1}{\left(\lambda_{j, k}{ }^{U}-\lambda_{j, k}{ }^{D}\right)}\right), \quad j=1,2, \cdots, m
$$

3) Sometimes, a little opinion about index weight given by some evaluators is precision value, while the rest is interval value. At this time, we might as well suppose that the number of evaluators who give precision value to index weight is $\mathrm{p}$ and the number of evaluators who give interval value to index weight is $q$, then $p+q=L$. Now we can obtain the bargaining weight named $\lambda_{j}^{\prime \prime}$ for the evaluators who give interval value to index weight by last method, then the bargaining weight named $\lambda_{j}^{\prime}$ can be determined, it satisfies the equation that $\lambda_{j}^{\prime}=\lambda_{j}^{\prime \prime}+\sum_{k=1}^{p} \eta_{k} \lambda_{j, k}$.After the normalization processing $\lambda_{j}=\lambda_{j}^{\prime} / \sum_{j=1}^{m} \lambda_{j}^{\prime}$, the final bargaining weight named $\lambda_{j}$ can be obtained.

\section{Application case}

Choose the case about fighter purchase from literature [10]. In the case there are six evaluation index which are largest speed (named $x_{1}$ ), farthest flight distance (named $x_{2}$ ), largest weight (named $x_{3}$ ), purchase price (named $x_{4}$ ), reliability (named $x_{5}$ ), sensitivity (named $x_{6}$ ), the index data is shown in table 1. Ten experts in the field were invited to solve the problem. Due to the objectivity of the evaluation data, so there is no sense to bargain with the index value. But the evaluators have different background (influence information under the view of every evaluator is shown in table 2 and table 3 ), and they have different opinions about index weight(relevant information of index weight is shown in table 4), then they decided to bargaining with index weight for determining the final purchase plan.

Table 1 Index value of each plan

\begin{tabular}{ccccccc}
\hline & $x_{1}$ & $x_{2}$ & $x_{3}$ & $x_{4}$ & $x_{5}$ & $x_{6}$ \\
\hline$o_{1}$ & 2.0 & 1500 & 20000 & 5.5 & 5 & 9 \\
$o_{2}$ & 2.5 & 2700 & 18000 & 6.5 & 3 & 9 \\
$o_{3}$ & 1.8 & 2000 & 21000 & 4.5 & 7 & 7 \\
$o_{4}$ & 2.2 & 1800 & 20000 & 5.0 & 5 & 5 \\
$o_{5}$ & 2.1 & 2100 & 19750 & 5.3 & 6 & 6 \\
$o_{6}$ & 2.3 & 2300 & 20800 & 5.2 & 6 & 8 \\
\hline
\end{tabular}

Table 2 Influence sorting under the view of every evaluator

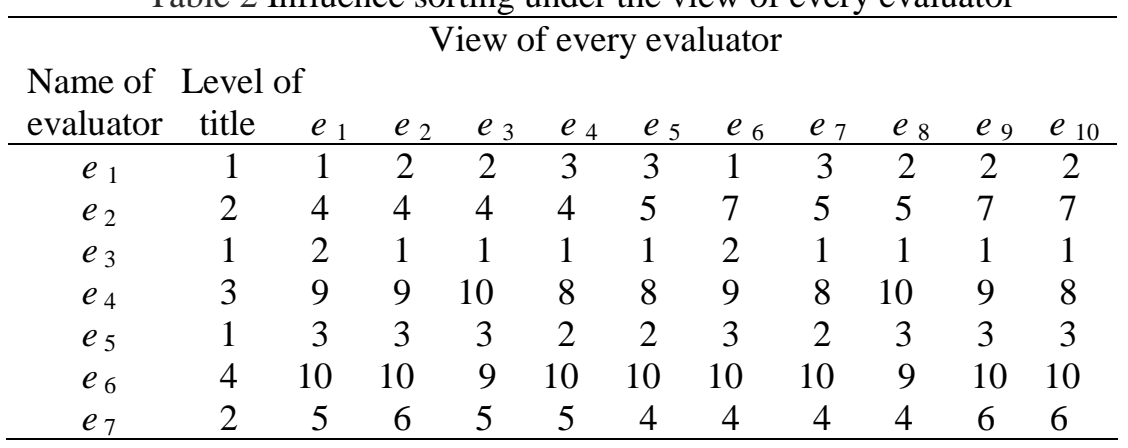




\begin{tabular}{llllllllllll}
\hline$e_{8}$ & 3 & 8 & 8 & 7 & 9 & 9 & 8 & 9 & 8 & 8 & 9 \\
$e_{9}$ & 2 & 6 & 5 & 6 & 7 & 6 & 5 & 6 & 7 & 4 & 5 \\
$e_{10}$ & 2 & 7 & 7 & 8 & 6 & 7 & 6 & 7 & 6 & 5 & 4
\end{tabular}

Table 3 Influence sorting under the view of every evaluator

\begin{tabular}{ccccccccccc}
\hline $\begin{array}{c}\text { Ratio of } \\
\text { influence }\end{array}$ & $e_{1}$ & $e_{2}$ & $e_{3}$ & $e_{4}$ & $e_{5}$ & $e_{6}$ & $e_{7}$ & $e_{8}$ & $e_{9}$ & $e_{10}$ \\
\hline$r_{2}$ & 1.4 & 1.2 & 1.4 & 1.4 & 1.6 & 1.2 & 1.2 & 1.2 & 1.8 & 1.2 \\
$r_{3}$ & 1.2 & 1.6 & 1.2 & 1.2 & 1.2 & 1.4 & 1.6 & 1.4 & 1.2 & 1.8 \\
$r_{4}$ & 1.4 & 1.2 & 1.6 & 1.2 & 1.6 & 1.2 & 1.2 & 1.6 & 1.4 & 1.2 \\
$r_{5}$ & 1.6 & 1.4 & 1.2 & 1.8 & 1.4 & 1.2 & 1.8 & 1.4 & 1.2 & 1.2 \\
$r_{6}$ & 1.4 & 1.4 & 1.8 & 1.4 & 1.6 & 1.6 & 1.2 & 1.2 & 1.2 & 1.4 \\
$r_{7}$ & 1.2 & 1.2 & 1.2 & 1.2 & 1.8 & 1.2 & 1.2 & 1.4 & 1.6 & 1.2 \\
$r_{8}$ & 1.4 & 1.6 & 1.2 & 1.6 & 1.6 & 1.8 & 1.4 & 1.6 & 1.2 & 1.4 \\
$r_{9}$ & 1.8 & 1.8 & 1.4 & 1.2 & 1.2 & 1.2 & 1.8 & 1.4 & 1.4 & 1.6 \\
$r_{10}$ & 1.4 & 1.2 & 1.2 & 1.4 & 1.8 & 1.4 & 1.2 & 1.4 & 1.2 & 1.2 \\
\hline
\end{tabular}

Table 4 The weight intervals which are given by each evaluator

\begin{tabular}{ccccccc}
\hline \multicolumn{7}{c}{ Evaluation index } \\
\multicolumn{8}{c}{ Evaluator } & $x_{1}$ & $x_{2}$ & $x_{3}$ & $x_{4}$ & $x_{5}$ & $x_{6}$ \\
\hline$e_{1}$ & {$[0.10,0.35]$} & {$[0.20,0.30]$} & {$[0.15,0.20]$} & {$[0.10,0.15]$} & {$[0.10,0.25]$} & {$[0.15,0.20]$} \\
$e_{2}$ & {$[0.20,0.30]$} & {$[0.20,0.30]$} & {$[0.10,0.35]$} & {$[0.25,0.30]$} & {$[0.05,0.25]$} & {$[0.15,0.20]$} \\
$e_{3}$ & {$[0.10,0.35]$} & {$[0.20,0.30]$} & {$[0.15,0.20]$} & {$[0.10,0.15]$} & {$[0.05,0.25]$} & {$[0.15,0.20]$} \\
$e_{4}$ & {$[0.10,0.15]$} & {$[0.25,0.30]$} & {$[0.15,0.20]$} & {$[0.15,0.30]$} & {$[0.10,0.25]$} & {$[0.05,0.30]$} \\
$e_{5}$ & {$[0.10,0.15]$} & {$[0.20,0.30]$} & {$[0.10,0.35]$} & {$[0.15,0.20]$} & {$[0.05,0.15]$} & {$[0.15,0.20]$} \\
$e_{6}$ & {$[0.20,0.30]$} & {$[0.20,0.30]$} & {$[0.15,0.20]$} & {$[0.10,0.15]$} & {$[0.10,0.25]$} & {$[0.05,0.30]$} \\
$e_{7}$ & {$[0.10,0.35]$} & {$[0.20,0.30]$} & {$[0.10,0.35]$} & {$[0.15,0.20]$} & {$[0.10,0.25]$} & {$[0.15,0.20]$} \\
$e_{8}$ & {$[0.10,0.15]$} & {$[0.20,0.30]$} & {$[0.10,0.35]$} & {$[0.20,0.30]$} & {$[0.05,0.25]$} & {$[0.10,0.15]$} \\
$e_{9}$ & {$[0.10,0.25]$} & {$[0.25,0.35]$} & {$[0.20,0.35]$} & {$[0.10,0.35]$} & {$[0.05,0.15]$} & {$[0.05,0.30]$} \\
$e_{10}$ & {$[0.20,0.30]$} & {$[0.20,0.30]$} & {$[0.10,0.35]$} & {$[0.15,0.20]$} & {$[0.10,0.25]$} & {$[0.15,0.20]$} \\
\hline
\end{tabular}

The solving process of the problem is listed as follows:

Step 1: Normalize each index data by use the extreme processing method which is proposed from literature [10], and the data can be expressed by matrix $\boldsymbol{A}$.

$$
\boldsymbol{A}=\left[\begin{array}{rrrrrr}
0.286 & 0 & 0.667 & 0.500 & 0.500 & 1.000 \\
1.000 & 1.000 & 0 & 0 & 0 & 1.000 \\
0 & 0.417 & 1.000 & 1.000 & 1.000 & 0.500 \\
0.571 & 0.250 & 0.667 & 0.750 & 0.500 & 0 \\
0.429 & 0.500 & 0.583 & 0.600 & 0.750 & 0.250 \\
0.714 & 0.667 & 0.933 & 0.650 & 0.750 & 0.750
\end{array}\right]
$$

Step 2: Calculate the evaluators' influence. By using the method given in section A, the evaluators' influence can be obtained. The calculation result is as follow, $\eta_{1}=1.868, \eta_{2}=0.771$, $\eta_{3}=2.455, \quad \eta_{4}=0.212, \quad \eta_{5}=1.536, \quad \eta_{6}=0.153, \quad \eta_{7}=0.796, \quad \eta_{8}=0.251, \quad \eta_{9}=0.601, \quad \eta_{10}=0.510$.

Step 3: Calculate the bargaining weight. By using the method given in section A, the bargaining weight can be obtained. The calculation result is as follow, $\lambda_{1}=0.124, \lambda_{2}=0.210, \lambda_{3}=0.191$, $\lambda_{4}=0.163, \lambda_{5}=0.126, \lambda_{6}=0.186$.

Step 4: Calculate the evaluation result. By using the information gathering method named weighted arithmetic average method, the evaluation result is as follow, $y_{1}=0.493, y_{2}=0.520$, 
$y_{3}=0.660, \quad y_{4}=0.436, \quad y_{5}=0.508, \quad y_{6}=0.747$, so the ranking of flight purchase plan is $o_{6} \succ o_{3} \succ o_{5} \succ o_{2} \succ o_{1} \succ o_{4}$.

\section{Conclusion}

In response to the characteristics of the bargaining evaluation, we provides a method which is used to deal with the large group bargaining evaluation problem, this method has the following features:

First, traditional methods relies too much on consistency degree of judgment matrix to determine evaluators' influence, but a high consistency degree of judgment matrix occasionally doesn't mean the conclusion is reliable, so a method based on rank correlation analysis method I (G1-) is proposed to determine evaluators' dynamic influence.

Second, the method given by literature [8] is limited by assumptions, but the method which is proposed in the paper is more simple, so it may has widely use in actual life.

Third, the method not only can help evaluation demander choose proper scale evaluators for evaluation problem, but also help public judge the credibility of evaluation conclusion based on the evaluators' influence.

In view the universality of bargaining evaluation model, many problems remains to be further discussed.

\section{References}

[1] Y. J. Guo. Discussion about bargaining evaluation method. Decision and decision support system, (1993)No. 4, p.100 106.

[2] J. X. Liu and Q. Fei. Method of determining attribute weight for bargaining evaluation problem. Control and decision, Vol.20(2005)No.6, p.713-716.

[3] W. W. Li, Y. J. Guo, P. T. Yi and Z. Y. He. An objective self-determine comprehensive evaluation method based on collaborative perspective. Journal of systems \& management, Vol.21(2012)No.3, p.378-383.

[4] X. Chen and Z. P. Fan. Study on the assessment level of experts based onlinguistic assessment matrices. Journal of Systems Engineering, Vol.24(2006)No.1, p.111-115.

[5] X. Chen and Z. P. Fan. Study on assessment level of experts based on difference preference information. Systems Engineering Theory \& Practice, Vol.27(2007)No.2, p.27-35.

[6] Z. S. Xu and X. Q. Cai. Nonlinear optimization models for multiple attribute group decision making with intuitionistic fuzzy information. International Journal of International Journal of Intelligent Systems, Vol.25(2010)No.6, p.489-513.

[7] F. M. Zhang, Y. J. Guo and T. P. Yi. A based on monte carlo simulation group consultation evaluation method and its application, Operations Research and Management Science. Vol.19(2010)No.2, p.63-67.

[8] Q. Mao, Y. J. Guo and Y. Qi. Calculation method of evaluators' authority in group evaluation. Journal of Northeastern University(Natural Science), Vol.34(2012)No.2, p.297-300.

[9] Q. Mao and Y. J. Guo. Calculation method of evaluators' dynamic influence on bargaining evaluation. Operations Research and Management Science, Vol.23(2014)No.5, p.192-197.

[10] Y. J. Guo: Theory, Method and Application of Comprehensive Evaluation(Science Press, China 2007). 